\title{
Von der (un)geliebten Hygiene bis zur Personalentwicklung \\ 3. Seminar in Hamburg im Haus Rissen
}

Mit einem Teilnehmerrekord ging das fnb-Seminar im Haus Rissen in Hamburg zum dritten Mal über die Bühne. Der inhaltliche Schwerpunkt lag auf den beiden großen Themenblöcken Hygiene und Personalentwicklung. Dabei reichten die Themen von der Händehygiene der Pflegekräfte bis zum Umgang mit resistenten Erregern und von den Problemen der Personalbedarfsplanung bis zum Thema Weiterbildung in der nephrologischen Pflege.

Es war schon überwältigend - so viele Anmeldungen und Teilnehmer gab es noch nie. Einerseits macht uns das stolz, andererseits tun uns die Teilnehmer leid, die wieder abreisen mussten oder zum ersten Mal nicht an dem Seminar teilnehmen konnten. Um diese Unannehmlichkeiten in der Zukunft zu vermeiden, bitten wir die Anmeldungen für das Hamburgseminar im Haus Rissen 2009 spätestens am 21. Februar 2009 einzureichen.

\section{Hygiene von A bis Z}

Im Fokus stand zunächst die Verleihung des nephrologischen Pflegepreises 2007. Christa Tast überreichte ihn an den Fachweiterbildungskurs 5 der Weiterbildungsstätte der Kreiskliniken Traunstein-Trostberg GmbH. Petra Dörfler, Kerstin Gerpheide, Hedwig Kallsperger, Claudia Kretzschmar, Karin Losbichler, Marion Meier, Mario Peuser, Daniela Siebentritt und Andrea Schmidtbauer setzten sich mit dem Thema „Der Beginn des Pflegeprozesses zur ersten Hämodialyse ist zu spät" auseinander.

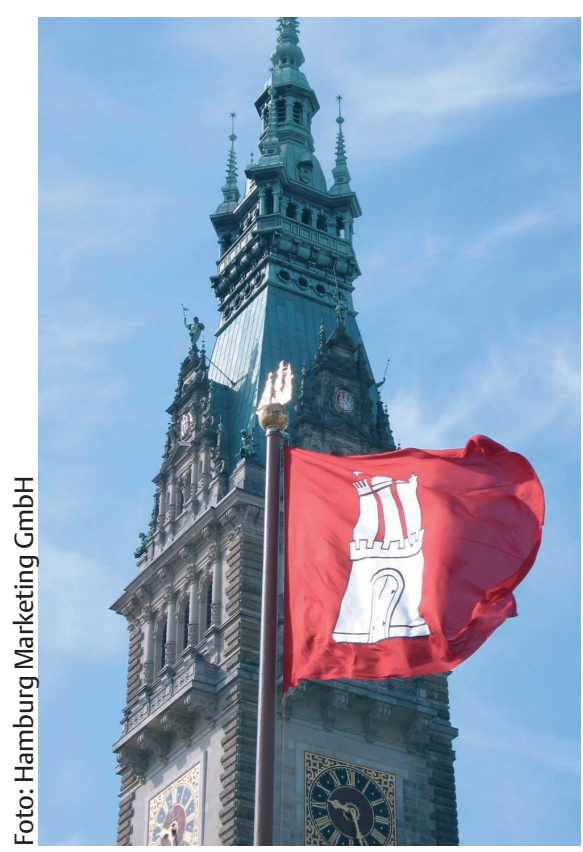

Nach einleitenden Worten von Josefa Fenselau verstand es Dipl.-Biol. Jasmine Dischinger, Bonn, in ihrem interessanten und anschaulichen Vortrag, allen Teilnehmern die Grundlagen der modernen Hygiene vor Augen zu führen. In der Kaffeepause konnten die Teilnehmer die persönliche Händehygiene apparativ überprüfen. Die Ergebnisse waren überraschend, die Schwachstellen deutlich zu sehen - auf jeden Fall gut zu wissen, denn schließlich ist medizinisches Personal der Hauptüberträger krankenhausspezifischer Infektionen. Dipl.-Biol. Heike Hohenadel vom KfH Neu-Isenburg, zeigte mit Ihrem Bildervortrag zum Thema „Infektionsquellen und Hygienerisiken im Dialysealltag“ sehr eindringlich die Fehler und die 
Schwachstellen in jeder Dialyseeinheit auf. Ihr zweiter Vortrag „Hygienemaßnahmen beim Umgang mit Patienten mit resistenten Erregern" gab Anlass zu einer regen Diskussion, in der alle Teilnehmer die Gelegenheit nutzten, ihre Fragen zu diesem Thema zu klären. Wieder einmal wurde deutlich, wie viel Klärungsbedarf besteht, doch letztlich bleiben immer wieder Fragen offen, da gesetzlich eindeutige Vorgaben fehlen und dadurch die Umsetzung im multidisziplinären Team zur täglichen Gradwanderung wird.

Udo Hentschke, Trainer der B. Braun Melsungen AG, stellte zum Schluss noch die gängigen Desinfektions- und Sterilisationsverfahren in seinem Vortrag „Grundlagen der Keimtötung, Resistenzstufen, Zielsetzung der Desinfektion und Desinfektionswirkstoffe" vor und rundete damit den Themenkreis ab. Der erste Tag fand in der dritten Jahreshauptversammlung des fnb seinen Abschluss. Der fnb dokumentierte hierin die erfolgreiche Arbeit der letzten drei Jahre. Die Mitgliederzahl steigt stetig an und der Verband arbeitet aktiv in vielen nationa-

\section{Ziel ist eine individuelle und trans- parente Personalbedarfsplanung}

Nach einem gemütlichen Abend mit viel Fachsimpelei und noch mehr Spaß, trafen sich am nächsten Morgen ein paar ganz "harte Teilnehmer“ zum mittlerweile fest etablierten „early morning run“.

Danach führte Jutta Balhorn die Teilnehmer in das Motto „Personalentwicklung: Erziehungsprogramm durch $\mathrm{Ar}$ beitgeber, Persönlichkeitsentfaltung für Arbeitnehmer, Produktionsmittelplalen und internationalen Projekten mit.

nung oder was ganz anderes?" in den zweiten Tag ein. Wie Herr Ückert (PHV Bad Homburg) anhand der quantitativen und qualitativen Personalbemessung zeigen konnte, haben sich die Zeiten doch sehr geändert und das Bemessungsschema ist mit der heutigen Personalstruktur nicht mehr zu realisieren. Nach dem Dialysestandard von 2006 sollte sich das heutige Dialyseteam zu $42 \%$ aus Pflegekräften, zu 35\% aus Fachpflegekräften und zu 25\% aus Arzthelferinnen zusammensetzen. Die Realität zeigt etwas anderes, die Einführung der Dialysepauschale, die steigende Multimorbidität der Dialysepatienten, alte räumliche Strukturen und steigende administrative Aufgaben machen eine qualitativ-einheitliche Versorgung der Patienten im Dialysealltag fast unmöglich.

Eine individuelle und transparente Personalbedarfsplanung ist deshalb unbedingt notwendig. Doch wie ist das finanzierbar? Dr. Claus Clasen, Prokurist der Kreiskliniken-Trostberg $\mathrm{GmbH}$ antwortete auf diese Frage mit seiner Hypothese „Ohne Teamarbeit geht es nicht, denn der Fisch stinkt immer vom Kopf her". Finanzierbar ist es, wenn Qualität bezahlt und altes Säulendenken abgeschafft wird. Mit regelmäßigen Zielvorgaben und Erfolgskontrollen sind Krankenhäuser und Dialyseeinrichtungen in Zukunft wieder wirtschaftlich zu führen. Systematische Personalentwicklung wie transparente Personalbedarfsplanung, leistungsorientierte Bezahlung, strukturierte Einarbeitung mit „Kümmerern“ für neue Mitarbeiter, Perspektiven und Weiterbildungen schafft die Identifikation der Mitarbeiter mit dem Unternehmen und fördert konstruktive Mitarbeit.

\section{Unklarheiten beseitigen}

Entgegen der Darstellung der AfnP in der letzten Ausgabe der Dialyse aktuell vertritt der Vorstand des fnb auch weiterhin einstimmig die Meinung, dass ein gemeinsamer Pflegeverband für die nephrologische Pflege in Deutschland sinnvoll und richtig ist. Die gemeinsamen Fusionsverhandlungen zwischen AfnP und fnb wurden am 21.02.2006 durch Vertreter des damaligen Vorstandes der AfnP abgebrochen und nicht, wie behauptet, durch den fnb. Sollte die Entscheidung des derzeitigen AfnP-Vorstandes zum Abbruch der Fusionsverhandlungen auf der Grundlage dieser Falschinformationen entstanden sein, so freuen wir uns, wenn wir dieses Missverständnis klären und gemeinsam mit dem AfnP-Vorstand an den Verhandlungstisch zurückkehren können. Bei dieser Gelegenheit möchten wir auch die dargestellte Zusammenstellung der nephrologischen Pflegeverbände in Deutschland korrigieren: Seit dem 1.1.2006 gibt es keinen Deutschen Zweig der EDTNA/ERCA mehr.

Thomas Fernsebner und Christa Tast für den Vorstand des fnb
Zum Schluss attestierte Clasen der Pflege, dass sie in dieser Umsetzung den Ärzten und der Verwaltung einen weiten Schritt voraus ist. Die anschließende Diskussion zeigte den Unmut und die Unzufriedenheit der Teilnehmer über die wachsende Arbeitsbelastung und die mangelnde Anerkennung ihrer Profession.

\section{Lebenslanges Lernen ist Aufgabe der Pflege}

Dietmar Wiederhold (Institut für Nephrologische Fort- und Weiterbildung Hann. Münden) befasste sich in seinem Vortrag „Was jeder gut findet, aber nicht alle tun: Fortbildung von Pflegekräften“ mit einer Befragung von Pflegekräften zum Thema Weiterbildung. Danach interessieren sich Pflegekräfte mehr für Pflegetechniken und Praxisthemen als für Management, Gesprächsführung und ähnliche Themen. Weiterbildungen sollten sich mehr an gefragten Themen und der Praxis orientieren und bessere Rahmenbedingungen und neue pädagogische Formen schaffen. Sein Fazit: Lebenslanges Lernen ist Aufgabe der Pflege, um damit auf den kontinuierlichen Wandel in der Berufswirklichkeit reagieren zu können. Durch gezielte Personalentwicklung mit praxisnahen Weiterbildungsangeboten und neuen Lernstrukturen kann sich die Pflege den steigenden Anforderungen, den kurzen Halbwertszeiten medizinischen Wissens und den Qualitätsanforderungen stellen. Die Pflegepädagogin Carola Walter (Kreiskliniken Traunstein Trostberg $\mathrm{GmbH}$ ) stellte in ihrem Beitrag „Wenn Moses nicht zum Berg kommt" passend dazu neue Lernformen für Fort- und Weiterbildungen vor. Resümee dieses Vortrages war es, dass E-Learning am Computer ohne Präsenzzeiten für Anwender wie Pflegepersonal in Vollzeitarbeit, eine Möglichkeit der individuellen Fort- und Weiterbildung schaffen. Es ermöglicht ein individuelles Lerntempo und Flexibilität. Für die Anbieter und Institutionen der Fort- und Weiterbildungen sorgt es für Kosteneinsparung, verkürzte Präsenszeiten, Zusammenarbeit zwischen Praxis und Theorie und eine schnelle Problembearbeitung via E-Mail. Auch die Diskussion nach diesem Beitrag machte klar, dass trotz aller Angebote nicht alle Pflegende ins Boot zu holen sind, aber im Haus Rissen schon eine starke Mannschaft an Bord war.

Kerstin Gerpheide, Vorstand fnb 